Research Article

\title{
Driving Fatigue Detection from EEG Using a Modified PCANet Method
}

\author{
Yuliang Ma (iD, ${ }^{1}$ Bin Chen, ${ }^{1,2}$ Rihui Li, ${ }^{2}$ Chushan Wang, Jun Wang, ${ }^{3}$ Qingshan She ${ }^{3}$, ${ }^{1}$ \\ Zhizeng Luo $\left(\mathbb{0},{ }^{1}\right.$ and Yingchun Zhang $\mathbb{(}^{2}$ \\ ${ }^{1}$ Intelligent Control \& Robotics Institute, College of Automation, Hangzhou Dianzi University, Hangzhou, China \\ ${ }^{2}$ Department of Biomedical Engineering, University of Houston, Houston, Texas, USA \\ ${ }^{3}$ Guangdong Provincial Work Injury Rehabilitation Hospital, Guangzhou, China
}

Correspondence should be addressed to Yingchun Zhang; yzhang94@uh.edu

Received 11 February 2019; Revised 28 April 2019; Accepted 19 June 2019; Published 14 July 2019

Guest Editor: Sangtae Ahn

Copyright (c) 2019 Yuliang Ma et al. This is an open access article distributed under the Creative Commons Attribution License, which permits unrestricted use, distribution, and reproduction in any medium, provided the original work is properly cited.

The rapid development of the automotive industry has brought great convenience to our life, which also leads to a dramatic increase in the amount of traffic accidents. A large proportion of traffic accidents were caused by driving fatigue. EEG is considered as a direct, effective, and promising modality to detect driving fatigue. In this study, we presented a novel feature extraction strategy based on a deep learning model to achieve high classification accuracy and efficiency in using EEG for driving fatigue detection. EEG signals were recorded from six healthy volunteers in a simulated driving experiment. The feature extraction strategy was developed by integrating the principal component analysis (PCA) and a deep learning model called PCA network (PCANet). In particular, the principal component analysis (PCA) was used to preprocess EEG data to reduce its dimension in order to overcome the limitation of dimension explosion caused by PCANet, making this approach feasible for EEG-based driving fatigue detection. Results demonstrated high and robust performance of the proposed modified PCANet method with classification accuracy up to 95\%, which outperformed the conventional feature extraction strategies in the field. We also identified that the parietal and occipital lobes of the brain were strongly associated with driving fatigue. This is the first study, to the best of our knowledge, to practically apply the modified PCANet technique for EEG-based driving fatigue detection.

\section{Introduction}

As a leading factor in traffic accidents, driving fatigue accounts for $14 \%-20 \%$ of motor vehicle accidents that cause serious injuries and fatalities to human life [1]. Consequently, growing attention has been paid to driving safety in recent years. Driving safety is thought to be affected by multiple factors, including monotonous environments, sleep deprivation, chronic sleepiness, drug and alcohol use [2, 3], wherein the most common factor is driving fatigue $[4,5]$. In such situation, drivers are most likely to fall asleep and drive unconsciously, which is not only a serious threat to the driver's own life and safety, but also a serious threat to the involved pedestrians and other vehicles. Therefore, detection of driving fatigue is of great importance to reduce the frequency and severity of traffic accidents [6].
In recent years, a variety of methods have been proposed to detect driving fatigue. For instance, Hiesh and Tai developed an infrared light-based digital signal processing (DSP) embedded system to capture driver's face and detect driving fatigue by identifying the opening and closing of eyes [7]. In another study, a calculation method named as the improved percentage of eyelid closure over the pupil overtime (PERCLOS) was employed as a standard criteria to judge whether the driver was tired or not [8]. Although this method is convenient in fatigue detection, it is vulnerable to environmental illumination such as the brightness, resulting in poor detection performance [9]. Later on, a sensor-based device called the steering wheel monitoring system (SAM) was developed to detect driving fatigue by monitoring the abnormal movement of the vehicle's steering wheel [10]. Although detection of steering wheel features good 
real-time performance and low cost, it also suffers poor antiinterference ability and low reliability [11].

Recently, fatigue detection based on physiological signals such as electroencephalogram (EEG), electrocardiogram (EOG), electromyogram (EMG), and electrocardiogram (ECG) signals has been increasingly investigated in this field [4, 12-19]. Among them, EEG has some major assets in detecting driving fatigue due to its high temporal resolution, high portability, and good sensitivity to fatigue. With this in mind, a variety of studies have attempted to perform EEGbased classification using different signal processing techniques to accurately detect the fatigue during driving. For instance, Yang et al. implemented the detection of driving fatigue using information fusion and dynamic Bayesian neural network [20]. In another study, Zhao et al. demonstrated that wavelet packet can be used to extract features from EEG signal and classify the driving condition by a support vector machine (SVM) [21]. However, the EEGbased fatigue detection during driving is still faced with challenges. For instance, EEG signals are usually collected with low signal to noise ratio (SNR), which requires large effort in preprocessing raw EEG data. Moreover, the widely employed EEG-based classification techniques depend heavily on handcrafted features, which is time-consuming and highly relies on skilled person in the domain before predictions are performed. Therefore, there is a clear need to develop a new strategy to improve the robustness and efficiency of EEG-based driving fatigue detection.

As a simplified deep learning model based on convolution neural network (CNN), principal component analysis network (PCANet) has been developed and widely used for feature extraction in two-dimensional image processing [22]. Referring to a previous study that performed EEGbased lie detection [23], PCANet was proved to be highly effective in classification problem as it automatically extracted features from multichannel EEG data based on the deep learning technique rather than extracting handcrafted features in conventional ways. However, PCANet may be subject to the phenomenon of dimension explosion when the dimensionality of input data is large, which dramatically increases the complexity and cost of computation, rendering it more difficult to be effectively employed in multichannel EEG signal processing.

To address this challenge, this study aimed to adapt the PCANet algorithm to enhance the efficiency of conventional EEG-based driving fatigue detection by incorporating the principal component analysis (PCA) with the PCANet technique. Specifically, PCA is used to preprocess the multichannel EEG signals and reduce the dimensionality of data prior to the PCANet processing. The performance of the proposed modified PCANet method in driving fatigue detection was evaluated by comparing to traditional PCANet and other conventional features extraction methods widely used in driving fatigue detection [4].

\section{Methods and Material}

2.1. General Structure and Purpose. The main structure of this study is demonstrated in Figure 1. EEG data was first

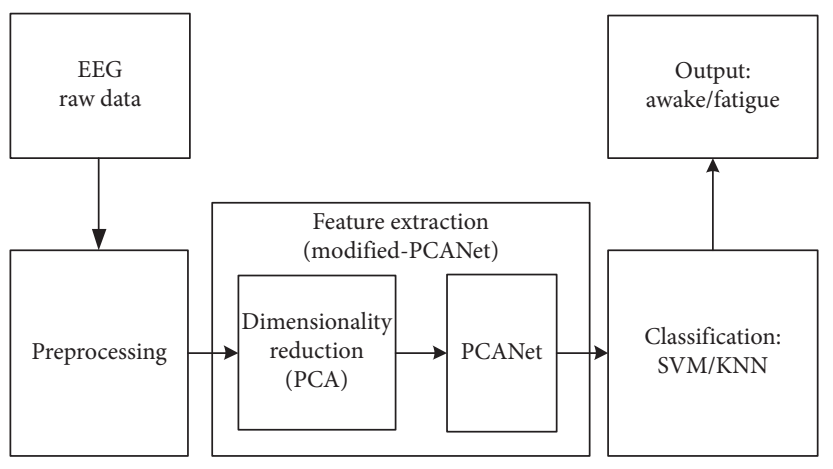

FIgURE 1: The overall schematic for the proposed EEG-based driving fatigue classification.

collected in a simulated driving environment, and followed by conventional signal preprocessing procedures. PCA was then used to reduce the dimensionality of the preprocessed signals, which were fed into the PCANet for feature extraction. Finally, the extracted features were classified using a support vector machine (SVM) and a K-Nearest Neighbors (KNN) classifier with respect to the driving condition.

2.2. Participants. Six male volunteers (right handed, aged $25.00 \pm 2.00$ years) with valid driver's licenses were recruited to participate in the experiment. All subjects were physically and psychologically healthy without any sleep disorders. The experiment was approved by the research ethics board of Guangdong Provincial Work Injury Rehabilitation Center and performed in accordance with the Declaration of Helsinki. Each subject was fully informed about the purpose of the research and provided written, informed consent prior to the start of the experiment.

\subsection{Experiment Design and Data Acquisition. A 32-channel} EEG acquisition system (Brain Products $\mathrm{GmbH}$, Germany) was utilized to collect EEG signals with the sampling frequency set to $500 \mathrm{~Hz}$. EEG electrodes were placed on the scalp according to the international 10-20 standard system. An advanced driving simulation system (Shanghai Infrared Automobile Simulator Driving Equipment Co., Ltd., China) was used in this study to simulate a real driving environment. Briefly, the driving simulation system could imitate the real driving scenarios with dynamically changing representations of the car and surrounding traffic. As shown in Figure 2, the driving simulation system consisted of a fixed car steering wheel, the brake and accelerator pedals, three large screens, a high-performance computer, driving simulation software, and a multifunctional data acquisition board. This system can be adapted to measure the EEG signal in different driving states in real time.

All participants were given sufficient time to practice and get familiar with the driving simulation system prior to the experiment. Datasets in two states were collected for each subject in this study, including the awake state and fatigue state. To collect the data in the awake state, all subjects were required to maintain a natural and adequate sleep for about 8 hours during the night before the experiment. EEG data 


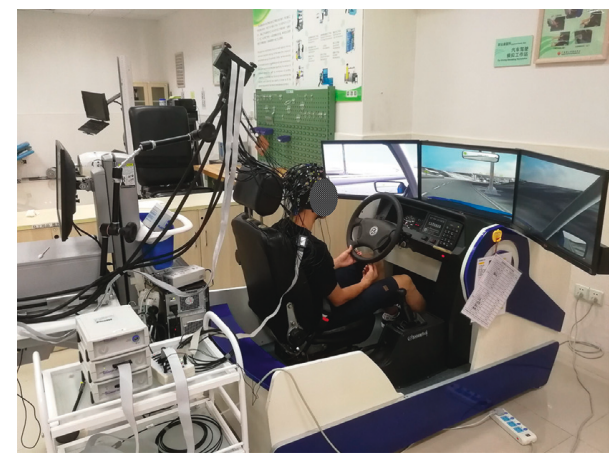

FIGURE 2: The setup of the experiment, including the driving platform and EEG recording system.

was collected at 9 a.m. on the next day for about 3060 minutes while subjects were driving in the simulated environment. To collect the data for the fatigue state, all subjects were allowed to sleep for only 4 hours during the night before the experiment. The EEG data was then measured at 9 a.m. for 30 to 60 minutes while subjects were driving in the simulated environment. Specifically, the driving path was set to relatively long, straight with smooth curves and almost no pedestrian to increase the drowsiness of the subjects in fatigue group, while the path was set to relatively complicated to avoid the drowsiness of the subjects in the awake group. During the recording, an observer seated 2 meters beside the subjects monitored the subject's behavior without causing any disturbance to the subjects. The observer decided whether the subject was in a fatigue state or an awake state by observing the subject's drowsy signs (more than two seconds eye closure and head nodding, large deviation off the road) $[4,24]$. Data recording was terminated 30 minutes after the subjects began to show fatigue signs. If the subject did not show any sign of drowsiness after 60 minutes of measurement, the experiment was terminated and data from the subject were excluded from further analysis. The experiment was conducted in a quiet, undisturbed laboratory with ambient temperatures around $22^{\circ} \mathrm{C}$.

2.4. Data Preprocessing and Analysis. All the data analyses in this study were implemented using MATLAB (2014a, MathWorks, Natick, Massachusetts). 20-minute EEG signals in each state were selected for analysis. The raw multichannel EEG signals were first downsampled from $500 \mathrm{~Hz}$ to $200 \mathrm{~Hz}$. A third-order bandpass filter $(0.1-45 \mathrm{~Hz})$ was then applied to remove artifacts such as slow drift, high-frequency noise, and powerline interference. The 20-minute preprocessed EEG data for each state were then segmented by a 10-second time window, resulting in 120 samples for each state and each subject. It is worthy of noting that in this study one sample was a two-dimension matrix (32 channels $\times 2000$ data points). Overall, a total of 1440 samples were obtained from all subjects for classification (720 samples for awake and 720 samples for fatigue).

2.5. PCA Dimensionality Reduction and Extraction of Main Features. In this study, the proposed modified PCANet consisted of two steps, PCA-based dimensionality reduction and PCANet-based feature extraction (Figure 1). To overcome the dimension explosion problem caused by conventional PCANet, PCA was first employed to reduce the dimensionality of each EEG sample. Briefly, for a given EEG sample data (32-channel $\times 2000$-point), the PCA transformed the data to 2000 linearly uncorrelated components known as principal components, ordered by the amount of variance of the original data that each component accounts for $[25,26]$. By keeping the first $r$ components with the largest variances and removing the remaining components, the size of original sample data could be reduced to 32 by $r$. In order to keep the original signal information as intact as possible, we kept the previous $r$ components that accounted for at least $99 \%$ of the original signal as threshold, which was 20 for all samples in this study. Therefore, size of each sample was reduced to 32 by 20 after PCA optimization, and the optimized EEG data was analyzed using PCANet for feature extraction in the next step.

2.6. PCANet-Based Feature Extraction. As we introduced previously, PCANet is widely used in 2-D image processing, such as face recognition [27]. In this study, each optimized EEG sample was treated as a 2 -D data matrix $(32 \times 20)$ and fed into PCANet for feature extraction.

The workflow of the PCANet network is shown in Figure 3, and details of the algorithm can be found in $[22,28]$. In brief, the PCANet consists of two PCA-based filtering layers and an output layer that includes processing of binary hashing and blockwise histogram.

Assume we have $N$ input samples after the EEG data preprocessing. Here, each EEG sample is treated as a twodimensional signal of size $m \times n$ (channel number $\times$ sample number). Given an input EEG sample $X_{i}$, a sliding window of size $k_{1} \times k_{2}$ is used to centralize the EEG sample by subtracting the mean value of each window's data from the corresponding window. Each centralized window is further vectorized into a single column, from which the input EEG sample is converted to a new 2-D matrix $\bar{X}_{i}$ consisting of all centralized windows. The same processing is applied to all input EEG samples $\left(\left\{I_{i}\right\}_{i=1}^{N}\right)$ to obtain the following data structure:

$$
X=\left[\bar{X}_{1}, \bar{X}_{2}, \ldots, \bar{X}_{N}\right] \varepsilon R^{k_{1} k_{2} \times N c},
$$

where $c=\left(m-k_{1}+1\right)\left(n-k_{2}+1\right)$ is the number of columns in $\bar{X}_{i}$.

The new vectorized matrix $X$ is then used to perform the PCA filtration in the first layer. Specifically, the covariance matrix of $X$, denoted as $X_{\text {cov }}=X X^{\mathrm{T}} / N c$, is computed and applied to select the eigenvectors corresponding to $L_{1}$ principal eigenvalues as PCA filters $W_{l}^{1}$. For the $i$ th EEG sample, the output of the first PCA layer is then given by the convolution of the input EEG sample and the PCA filters:

$$
I_{i}^{l}=\bar{I}_{i} * W_{l}^{1}, \quad i=1,2, \ldots, N \text { and } l=1,2, \ldots, L_{1},
$$

where $I_{i}^{l}$ denotes the $l$ th output of the $i$ th EEG sample and $\bar{I}_{i}$ is the zero-padded form of $I_{i}$ to ensure the same size of $I_{i}^{l}$ and $I_{i}$. 


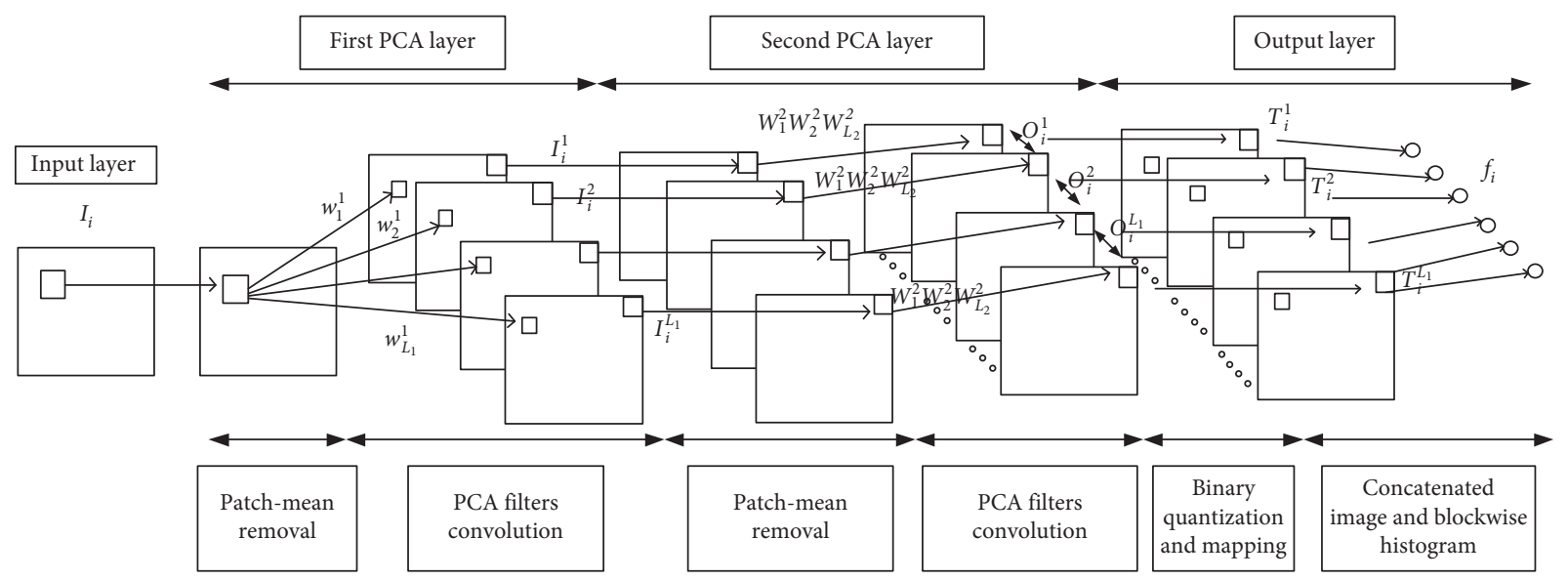

FIgURE 3: The PCANet network structure.

The second PCA layer is similar to the first layer. The output of the first PCA layer is centralized with the same sliding window and applied to select the eigenvectors corresponding to $L_{2}$ principal eigenvalues as PCA filters $W_{p}^{2}$. The output of the second PCA layer is given as

$$
O_{i}^{l} \doteq \bar{I}_{i}^{l} * W_{p}^{2}, \quad i=1,2, \ldots, N \text { and } p=1,2, \ldots L_{2} .
$$

With the result obtained from the filtrations of two PCA layers, the output of the PCANet $\left(T_{i}^{l}\right)$ is further processed by binary hashing as

$$
T_{i}^{l}=\sum_{p=1}^{L_{2}} 2^{p-1} H\left(\bar{I}_{i}^{l} * W_{p}^{2}\right), \quad l=1,2, \ldots, L_{1},
$$

where $H(\cdot)$ is a Heaviside step function that sets positive values as one and zero for others.

Finally, for the ith input EEG sample, each of the $L_{1}$ components in $T_{i}^{l}$ is partitioned into $B$ blocks. The histogram (with $2^{L_{2}}$ bins) of the decimal values in each block is computed and concatenated into one vector represented as Bhist $\left(T_{i}^{l}\right)$. The PCANet-derived feature of the $i$ th EEG sample is then denoted as

$$
f_{i}=\left[\operatorname{Bhist}\left(T_{i}^{1}\right), \ldots, \operatorname{Bhist}\left(T_{i}^{L_{1}}\right)\right]^{T} \in R^{\left(2^{L_{2}}\right) L_{1} B} .
$$

The PCANet processing is applied to each EEG sample for feature extraction.

2.7. Classification. Support vector machine (SVM) and $K$-Nearest Neighbors (KNN) were employed as classifiers in the classification of awake and fatigue states for each subject. The performance of each classifier was evaluated using a 10 -fold cross-validation strategy. At each iteration, $90 \%$ of the samples were randomly selected as the training set, and the accuracy, defined as the ratio between correct predictions and the total number of predictions, was computed on the remaining $10 \%$ of the samples, the testing set. Accuracies among the 10 steps of the cross validation were then averaged, yielding the mean accuracy for each subject. To evaluate the superiority of the proposed method, the obtained accuracy was compared to the performance obtained from the traditional PCANet method and two commonly used feature extraction methods, i.e., the power spectral density (PSD) $[29,30]$ and wavelet packet decomposition (WPD) [31].

In this study, the PSD features of each EEG channel in a segmented EEG sample (32-channel $\times 2000$-point) were estimated through Short-Time Fourier transform (STFT) with a 128-point Hanning window and 50\% overlap rate. For each EEG channel, the PSD feature of a specific frequency band was computed by averaging all squared magnitude values of STFT within the corresponding frequency range. In this study, five typical EEG bands were investigated, including delta $(0.1-4 \mathrm{~Hz})$, theta $(4-8 \mathrm{~Hz})$, alpha $(8-13 \mathrm{~Hz})$, beta $(13-20 \mathrm{~Hz})$, and gamma $(20-45 \mathrm{~Hz})$. This resulted in $160 \mathrm{PSD}$ features for each EEG sample (5-band $\times 32$-channel).

The WPD features of each EEG channel in a segmented EEG sample (32-channel $\times 2000$-point $)$ were calculated based on discrete wavelet decomposition (DWT). In brief, the DWT decomposed the selected EEG signal into a number of layers by filtering the signal with quadrature mirror filters (a low-pass filter and a high-pass filter). The output of each layer was a series of detail coefficients (from the high-pass filter) and approximation coefficients (from the low-pass filter), which were extracted as features for classification [32]. In this study, we decomposed each EEG channel data (2000 points) with a 3-layer "Daubechies" wavelet, resulting in 8 groups of coefficients ( 256 points). Therefore, there were in total 65536 (8-groups $\times 256$-point $\times 32$-channel) features extracted for each EEG sample.

\section{Results}

Referring to previous studies $[4,24]$, it was found that the alteration in brain regions during awake and fatigue states were more prominent at the parietal lobe at alpha and beta frequency bands $[33,34]$. Figure 4 shows the group-averaged PSD distribution of the relevant EEG signals in alpha (8$13 \mathrm{~Hz})$ and beta $(14-20 \mathrm{~Hz})$ bands in awake and fatigue states. The PSD values were computed based on the average of all 10-second EEG samples in each state for each subject. In brief, in the fatigue state, the PSD in the parietal and 


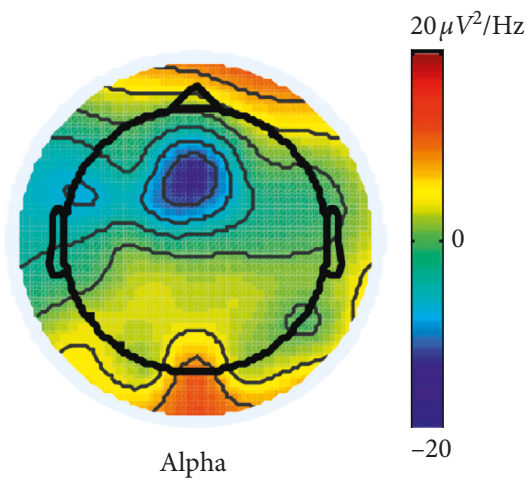

(a)

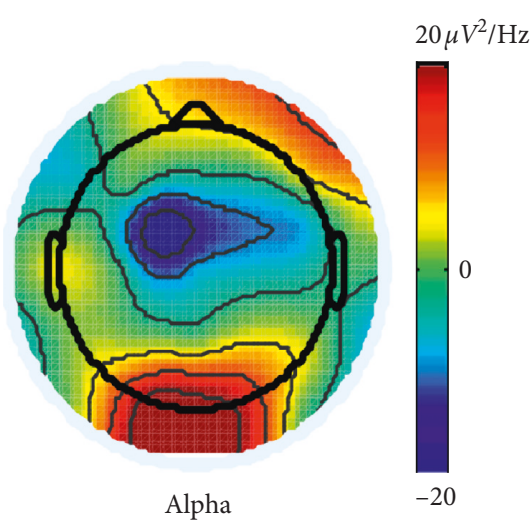

(c)

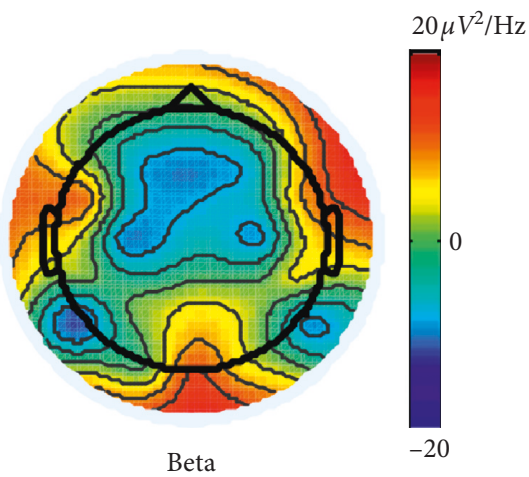

(b)

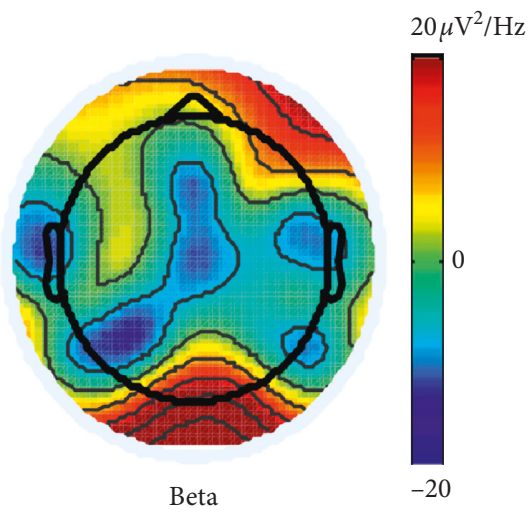

(d)

Figure 4: PSD distributions of alpha and beta bands for awake (a, b) and fatigue (c, d) states.

occipital lobes of the brain was more pronounced compared to the PSD distribution in the awake state.

In order to determine the optimal number of PCA filters $\left(L_{1}\right.$ and $\left.L_{2}\right)$ when using the PCANet, the classification performance varied with the number of PCA filters was acquired for each subject. Figure 5 shows the variation of classification performance for each subject when using a SVM, with number of PCA filters increasing from 2 to 14 for both layers, respectively. Overall, for most subjects (except sub. 3 and sub. 4), the classification performance was enhanced as the filter number increased, and gradually decreased when filter number was over 10 or 12 .

Additionally, the performance of classification using two classifiers and various feature extraction strategies, including the traditional PCANet, WPD, PSD, and the proposed modified PCANet method, is shown in Figure 6 and summarized in Tables 1 and 2. Overall, for both classifiers, when selecting the optimal PCA filter number for each individual subject, the traditional PCANet and the proposed modified PCANet method achieved better performance in the classification of awake and fatigue states. The result of the paired- $t$ test between four feature extraction methods revealed that the PCANet-based methods significantly outperformed the other two methods $(p<0.005)$, as shown in Figure 6 and Table 2. Although no significant difference in classification accuracy was observed

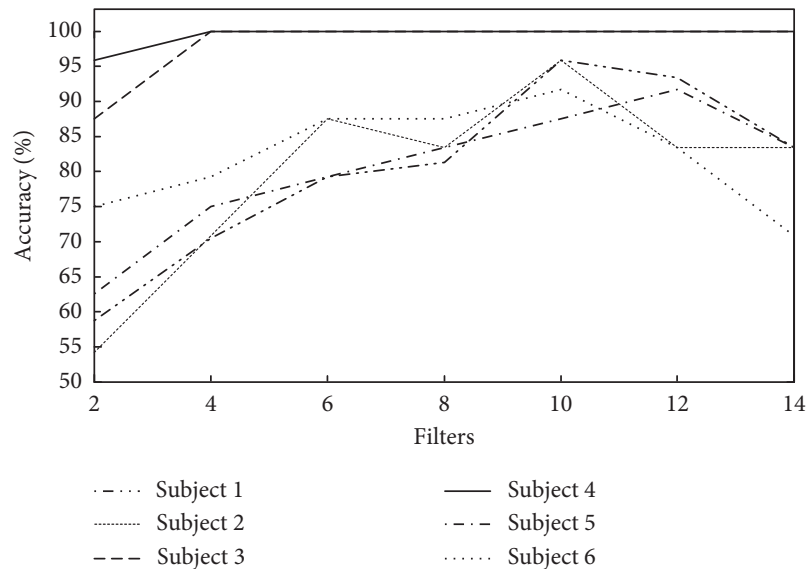

FIgURE 5: The classification performance of different PCA filter numbers when using SVM.

between the traditional PCANet and the modified PCANet, the time used in the feature extraction, model training, and testing was drastically reduced when using the modified PCANet method, indicating the high efficiency of this method (Table 3).

In addition, the area under the curve (AUC) of the receiver operating characteristic (ROC) curve [35], which evaluates how well a model separates the groups being classified, was employed to assess the performance of 


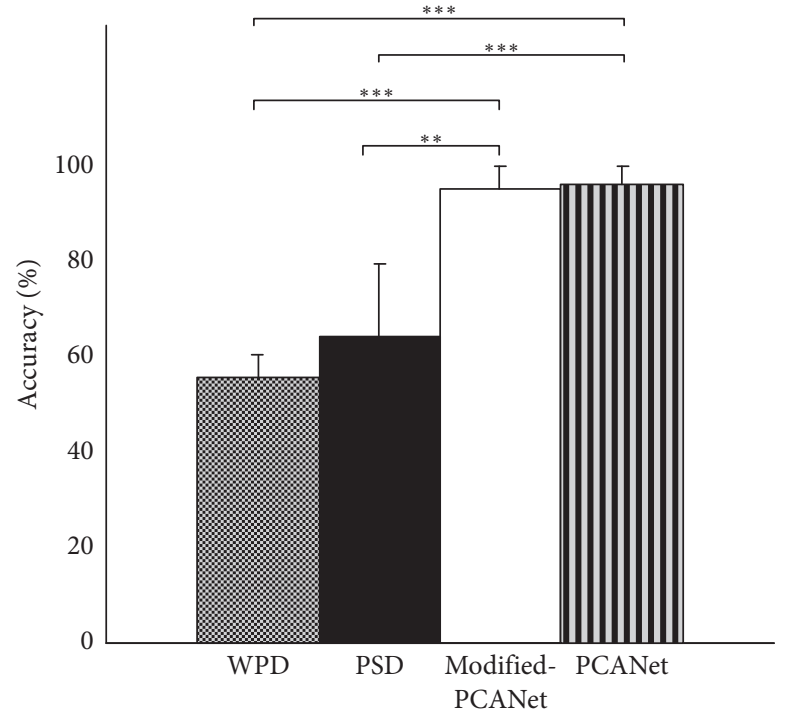

(a)

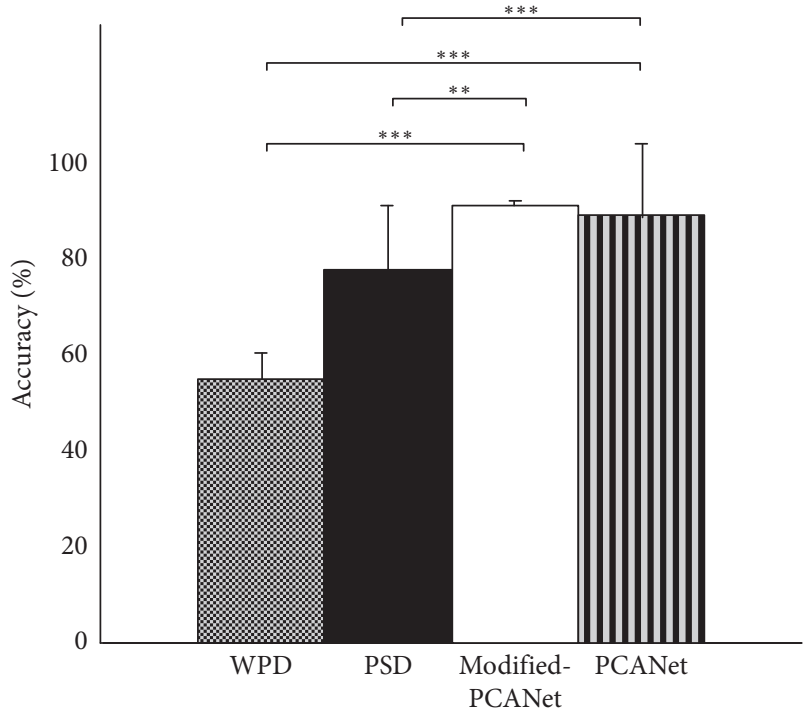

(b)

FIgURE 6: The average accuracies obtained by SVM (a) and KNN (b) when using different features extracted through WPD, PSD, modified PCANet, and PCANet. “**” denotes significantly different from controls $(p<0.005)$. “***” denotes significantly different from controls $(p<0.0001)$.

TABle 1: The classification performance using different feature extraction approaches.

\begin{tabular}{lccc}
\hline \multirow{2}{*}{ Methods } & Classifiers & \multicolumn{2}{c}{ Classification performance } \\
& & Accuracy (\%) & AUC \\
\hline \multirow{2}{*}{ WPD } & SVM & $55.42 \pm 5.09$ & $0.51 \pm 0.11$ \\
& KNN & $54.00 \pm 5.00$ & $0.46 \pm 0.08$ \\
\hline \multirow{2}{*}{ PSD } & SVM & $64.44 \pm 15.06$ & $0.55 \pm 0.12$ \\
& KNN & $76.00 \pm 13.00$ & $0.55 \pm 0.08$ \\
\multirow{2}{*}{ Modified-PCANet } & SVM & $95.14 \pm 4.87$ & $0.97 \pm 0.04$ \\
& KNN & $89.00 \pm 10.00$ & $0.89 \pm 0.12$ \\
\hline \multirow{2}{*}{ PCANet } & SVM & $96.00 \pm 4.00$ & $0.98 \pm 0.03$ \\
& KNN & $87.00 \pm 15.00$ & $0.91 \pm 0.10$ \\
\hline
\end{tabular}

TABLe 2: The summary of the statistical analysis ( $t$-test) of the classification performance between all feature extraction methods.

\begin{tabular}{lccc}
\hline \multirow{2}{*}{ Methods } & \multirow{2}{c}{ Classifiers } & \multicolumn{2}{c}{$p$ values } \\
& & Accuracy (\%) & AUC \\
\hline \multirow{2}{*}{ Modified-PCANet-WPD } & SVM & $1.36 e-07$ & $1.02 E-04$ \\
& KNN & $6.15 e-05$ & $5.18 E-04$ \\
\hline \multirow{2}{*}{ Modified-PCANet-PSD } & SVM & 0.0023 & $2.24 E-04$ \\
& KNN & 0.0018 & $3.10 E-03$ \\
\hline \multirow{2}{*}{ Modified-PCANet- } & SVM & 0.3541 & 0.6612 \\
PCANet & KNN & 0.6823 & 0.2884 \\
\hline
\end{tabular}

different feature extraction strategies. As summarized in Tables 1 and 2, for both classifiers, the AUC values obtained from the traditional PCANet and the proposed modified PCANet method are significantly higher compared to PSD and WPD features $(p<0.005)$. Similarly, no significant difference in AUC values was observed between the traditional PCANet and the modified PCANet.

\section{Discussion}

This study sought to validate the feasibility of using modified PCANet to enhance the performance of EEG-based driving fatigue detection. The neuronal electrical activity was recorded using EEG in a simulated driving environment with subjects experienced both awake and fatigue states. We employed PCA to alleviate the dimension explosion caused by PCANet before classification. The experimental results indicated a significantly enhanced performance in the fatigue detection compared to the traditional PCANet and other conventional approaches.

Alterations in low and high frequency bands were previously observed by EEG in the drowsy state [36]. In summary, investigations that included the transition from awake to sleepy states have demonstrated an increase in the alpha rhythm [24]. The alteration of the alpha band during drowsiness in both simulated and actual driving conditions was also reported in a previous study [37]. In this study, we compared the PSD between both states for alpha and beta frequency bands and found an increased PSD at occipital and parietal areas in both alpha and beta bands (Figure 4). This finding is in line with the results from these studies, demonstrating the possibility of using EEG as a portable and reliable approach to monitor and detect the driving fatigue.

In order to monitor the brain state during driving, it is of great importance to achieve high accuracy and reliability in detecting the driver's fatigue state. With high classification accuracy between the awake and fatigue states achieved by the modified PCANet approach, our study proved the usefulness of EEG to study driving fatigue. In particular, the substantial increase in classification accuracy using the proposed method, compared to conventional feature extraction methods, offers a new perspective to deal with classification problem when using multichannel biosignals 
TABLE 3: The average time (seconds) used in the feature extraction, model training, and testing between the traditional PCANet and the proposed modified PCANet method.

\begin{tabular}{|c|c|c|c|c|c|c|c|c|}
\hline \multirow{2}{*}{ Steps } & \multirow{2}{*}{ Methods } & & \multicolumn{6}{|c|}{ Number of PCA filters } \\
\hline & & & 2 & 4 & 6 & 8 & 10 & 12 \\
\hline \multirow{2}{*}{ Features extraction } & Modified-PCANet & & 0.89 & 1.46 & 2.18 & 2.91 & 4.22 & 7.71 \\
\hline & PCANet & & 125.43 & 219.94 & 320.39 & 449.50 & 651.95 & 1202.40 \\
\hline \multirow{4}{*}{ Model training } & \multirow{2}{*}{ Modified-PCANet } & SVM & 0.05 & 0.70 & 5.67 & 10.34 & 13.25 & 15.65 \\
\hline & & KNN & 0.02 & 0.30 & 2.20 & 4.10 & 5.12 & 6.15 \\
\hline & \multirow{2}{*}{ PCANet } & SVM & 7.02 & 126.66 & 302.52 & 736.79 & 1813.40 & 3606.80 \\
\hline & & KNN & 2.70 & 49.01 & 118.60 & 285.04 & 697.46 & 1387.23 \\
\hline \multirow{4}{*}{ Model testing } & \multirow{2}{*}{ Modified-PCANet } & SVM & 0.25 & 0.26 & 0.26 & 0.28 & 0.34 & 0.39 \\
\hline & & KNN & 0.10 & 0.12 & 0.13 & 0.15 & 0.13 & 0.16 \\
\hline & \multirow{2}{*}{ PCANet } & SVM & 1.16 & 2.94 & 5.34 & 11.10 & 20.84 & 35.75 \\
\hline & & KNN & 0.45 & 1.14 & 2.10 & 4.22 & 8.02 & 14.06 \\
\hline
\end{tabular}

such as EEG and EMG signals. It is noteworthy that PCA was necessarily adopted before PCANet was employed to extract features in this study. As shown in Table 3, the proposed modified PCANet method remarkably reduced the time for the classification while maintaining a comparable performance relative to the traditional PCANet approach. This provides evidence that PCA is able to alleviate the curse of dimensionality induced by PCANet, reducing the computational cost when using the traditional PCANet. By taking this great advantage, the proposed method is considered a more effective strategy in a practical scenario such as monitoring driving fatigue in real time. In addition, the components compressed by PCA retains the main characteristics of original signals, which is the inherent benefit offered by PCA. The pre-refined signals can be further improved by PCANet to achieve the significantly enhanced classification accuracy. Particularly, the classification performance across all subjects not only exhibited high accuracy, but also yielded lower variance, demonstrating the good robustness of the proposed method.

Despite the improvement achieved in this study, there are still several limitations in this study. Firstly, only off-line analysis and small sample size were elected in this study. Real-time fatigue classification should be conducted on larger population base in the future to validate the potential of the proposed approach in actual driving environment. In addition, two-layer PCA structure was applied for feature extraction in this study, and 8-12 filters were considered optimal setting for achieving best performance. Apparently, the number of PCA filters within each layer affected the quality of feature extraction, which significantly affected the performance and efficiency of the fatigue classification. Further investigation on how to automatically select the best filter number for each subject is required. Finally, in the present study we solely focused on adapting a deep learningbased technique to the conventional EEG-based driving fatigue classification and provide a new perspective to deal with classification problem when using multichannel biosignals. Although compelling result was achieved in current study, it is expected that future work will evaluate and employ the state-of-the-art algorithms to enhance the performance of this application. Even though the mentioned limitations may prevent us drawing a solid conclusion, the preliminary results demonstrate the capability of the proposed PCANet-based algorithm to monitor and detect the driving fatigue in advance so that it can prevent motor vehicle collision caused by driver drowsiness.

\section{Conclusion}

In this paper, a novel feature extraction strategy incorporating the PCA and PCANet techniques was proposed to enhance the classification performance in EEG-based driving fatigue detection. Significantly enhanced classification performance was achieved using the proposed modified PCANet method compared to the traditional PCANet algorithm and two conventional feature extraction strategies. Additionally, the power spectrum analysis of EEG signals indicated a higher power alteration at occipital and parietal areas in alpha and beta bands. The findings in this study not only demonstrated the effectiveness of using EEG to monitor driving fatigue but also provided a new perspective to adapt a novel machine learning algorithm to investigate the nature of philological signals.

\section{Data Availability}

The data used to support the findings of this study are available from the corresponding author upon request.

\section{Conflicts of Interest}

The authors declare that they have no conflicts of interest.

\section{Acknowledgments}

This work is supported in part by the National Natural Science Foundation of China (no. 61871427, 61671197, and 61372023) and the University of Houston.

\section{References}

[1] A. Fletcher, K. McCulloch, S. D. Baulk, and D. Dawson, "Countermeasures to driver fatigue: a review of public awareness campaigns and legal approaches," Australian \& New Zealand Journal of Public Health, vol. 29, no. 5, pp. 471-476, 2005. 
[2] P.-H. Ting, J.-R. Hwang, J.-L. Doong, and M.-C. Jeng, "Driver fatigue and highway driving: a simulator study," Physiology \& Behavior, vol. 94, no. 3, pp. 448-453, 2008.

[3] H. Summala, H. Hakkanen, T. Mikkola, and J. Sinkkonen, "Task effects on fatigue symptoms in overnight driving," Ergonomics, vol. 42, no. 6, pp. 798-806, 1999.

[4] S. Ahn, T. Nguyen, H. Jang, J. G. Kim, and S. C. Jun, "Exploring neuro-physiological correlates of drivers' mental fatigue caused by sleep deprivation using simultaneous EEG, ECG, and fNIRS data," Frontiers in Human Neuroscience, vol. 10, no. 848, 2016.

[5] G. Zhang, K. K. W. Yau, X. Zhang, and Y. Li, "Traffic accidents involving fatigue driving and their extent of casualties," Accident Analysis \& Prevention, vol. 87, pp. 34-42, 2016.

[6] H. Wang, C. Zhang, T. Shi, F. Wang, and S. Ma, "Real-time EEG-based detection of fatigue driving danger for accident prediction," International Journal of Neural Systems, vol. 25, no. 2, article 1550002, 2015.

[7] C.-S. Hsieh and C.-C. Tai, "An improved and portable eyeblink duration detection system to warn of driver fatigue," Instrumentation Science \& Technology, vol. 41, no. 5, pp. 429-444, 2013.

[8] J. J. Yan, H.-H. Kuo, Y.-F. Lin, and T.-L. Liao, "Real-time driver drowsiness detection system based on PERCLOS and grayscale image processing," in Proceedings of the International Symposium on Computer, Seoul, South Korea, June 2016.

[9] J. Jimenez-Pinto and M. Torres-Torriti, "Face salient points and eyes tracking for robust drowsiness detection," Robotica, vol. 30, no. 5, pp. 731-741, 2012.

[10] T. Åkerstedt, B. Peters, A. Anund, and G. Kecklund, "Impaired alertness and performance driving home from the night shift: a driving simulator study," Journal of Sleep Research, vol. 14, no. 1, pp. 17-20, 2010.

[11] A. Mittal, K. Kumar, S. Dhamija, and M. Kaur, "Head movement-based driver drowsiness detection: a review of stateof-art techniques," in Proceedings of the IEEE International Conference on Engineering \& Technology, Coimbatore, India, March 2016.

[12] R. Chai, G. R. Naik, T. N. Nguyen et al., "Driver fatigue classification with independent component by entropy rate bound minimization analysis in an EEG-based system," IEEE Journal of Biomedical and Health Informatics, vol. 21, no. 3, pp. 715-724, 2017.

[13] J. Hu, "Comparison of different features and classifiers for driver fatigue detection based on a single EEG channel," Computational and Mathematical Methods in Medicine, vol. 2017, Article ID 5109530, 9 pages, 2017.

[14] J. X. Ma, L. C. Shi, and B. L. Lu, "An EOG-based vigilance estimation method applied for driver fatigue detection," Neuroscience \& Biomedical Engineering, vol. 2, no. 1, pp. 4151, 2015.

[15] W. L. Zheng and B. L. Lu, "A multimodal approach to estimating vigilance using EEG and forehead EOG," Journal of Neural Engineering, vol. 14, no. 2, article 026017, 2017.

[16] L. Boon-Leng, L. Dae-Seok, and L. Boon-Giin, "Mobile-based wearable-type of driver fatigue detection by GSR and EMG," in Proceedings of the Tencon IEEE Region 10 Conference, Xi'an, China, January 2016.

[17] A. Tjolleng, K. Jung, W. Hong et al., "Classification of a driver's cognitive workload levels using artificial neural network on ECG signals," Applied Ergonomics, vol. 59, pp. 326-332, 2017.
[18] F. Wang, H. Wang, and R. Fu, "Real-time ECG-based detection of fatigue driving using sample entropy," Entropy, vol. 20, no. 3, p. 196, 2018.

[19] J. F. O'Hanlon and G. R. Kelley, “Comparison of performance and physiological changes between drivers who perform well and poorly during prolonged vehicular operation," in Vigilance, pp. 87-109, Springer, Boston, MA, USA, 1977.

[20] G. Yang, Y. Lin, and P. Bhattacharya, "A driver fatigue recognition model based on information fusion and dynamic Bayesian network," Information Sciences, vol. 180, no. 10, pp. 1942-1954, 2010.

[21] C. Zhao, C. Zheng, and M. Zhao, J. Liu, Y. Tu, "Automatic classification of driving mental fatigue with EEG by wavelet packet energy and KPCA-SVM," International Journal of Innovative Computing, Information and Control, vol. 7, no. 3, pp. 1157-1168, 2011.

[22] T.-H. Chan, K. Jia, S. Gao, J. Lu, Z. Zeng, and Y. Ma, "PCANet: a simple deep learning baseline for image classification?," IEEE Transactions on Image Processing, vol. 24, no. 12, pp. 5017-5032, 2015.

[23] L.-Y. Gu, W. Z. Lv, Y. Yang, J. F. Gao, J. A. Guan, and D. Zhou, "Deception detection study based on PCANet and support vector machine," Acta Electronica Sinica, vol. 44, no. 8, pp. 1969-1973, 2016.

[24] T. Nguyen, S. Ahn, H. Jang, S. C. Jun, and J. G. Kim, "Utilization of a combined EEG/NIRS system to predict driver drowsiness," Scientific Reports, vol. 7, no. 1, 2017.

[25] S. Poorna, V. V. Arsha, P. T. A. Aparna, P. Gopal, and G. J. Nair, "Drowsiness detection for safe driving using PCA EEG signals," in Progress in Computing, Analytics and Networking, pp. 419-428, Springer, Singapore, 2018.

[26] R. K Chaurasiya, N. D. Londhe, and S. Ghosh, "Statistical wavelet features, PCA, and SVM based approach for EEG signals classification," International Journal of Computer and Information Engineering, vol. 9, no. 2, pp. 182-186, 2015.

[27] L. Tian, C. Fan, and Y. Ming, "Multiple scales combined principle component analysis deep learning network for face recognition," Journal of Electronic Imaging, vol. 25, no. 2, article 023025, 2016.

[28] B. Li, Y. Dong, D. Zhao, Z. Wen, and L. Yang, "A PCANet based method for vehicle make recognition," in Proceedings of the 2016 IEEE 19th International Conference on Intelligent Transportation Systems (ITSC), IEEE, Rio de Janeiro, Brazil, November 2016.

[29] C. Kim, J. Sun, D. Liu, Q. Wang, and S. Paek, “An effective feature extraction method by power spectral density of EEG signal for 2-class motor imagery-based BCI," Medical \& Biological Engineering \& Computing, vol. 56, no. 9, pp. 16451658, 2018.

[30] N. G. Ozmen, L. Gumusel, and Y. Yang, "A biologically inspired approach to frequency domain feature extraction for EEG classification," Computational and Mathematical Methods in Medicine, vol. 2018, Article ID 9890132, 10 pages, 2018.

[31] Y. Zhang, B. Liu, X. Ji, and D. Huang, "Classification of EEG signals based on autoregressive model and wavelet packet decomposition," Neural Processing Letters, vol. 45, no. 2, pp. 365-378, 2017.

[32] R. Li, T. Potter, W. Huang, and Y. Zhang, "Enhancing performance of a hybrid EEG-fNIRS system using channel selection and early temporal features," Frontiers in Human Neuroscience, vol. 11, p. 462, 2017.

[33] J. P. Liu, C. Zhang, and C. X. Zheng, "Estimation of the cortical functional connectivity by directed transfer function 
during mental fatigue," Applied Ergonomics, vol. 42, no. 1, pp. 114-121, 2011.

[34] F. Gharagozlou, G. Nasl Saraji, A. Mazloumi et al., "Detecting driver mental fatigue based on EEG alpha power changes during simulated driving," Iranian Journal of Public Health, vol. 44, no. 12, pp. 1693-1700, 2015.

[35] T. Fawcett, "An introduction to ROC analysis," Pattern Recognition Letters, vol. 27, no. 8, pp. 861-874, 2006.

[36] I. Käthner, S. C. Wriessnegger, G. R. Müller-Putz, A. Kübler, and S. Halder, "Effects of mental workload and fatigue on the P300, alpha and theta band power during operation of an ERP (P300) brain-computer interface," Biological Psychology, vol. 102, no. 5, pp. 118-129, 2014.

[37] M. A. Schier, "Changes in EEG alpha power during simulated driving: a demonstration," International Journal of Psychophysiology, vol. 37, no. 2, pp. 155-162, 2000. 


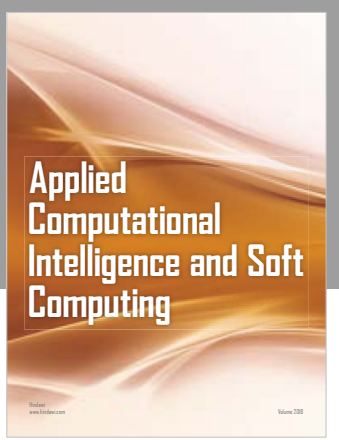

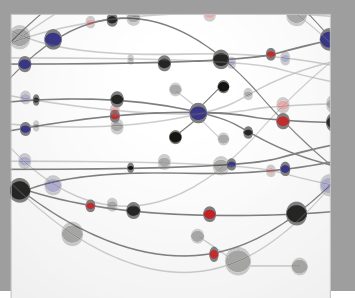

The Scientific World Journal
Submit your manuscripts at

Computing
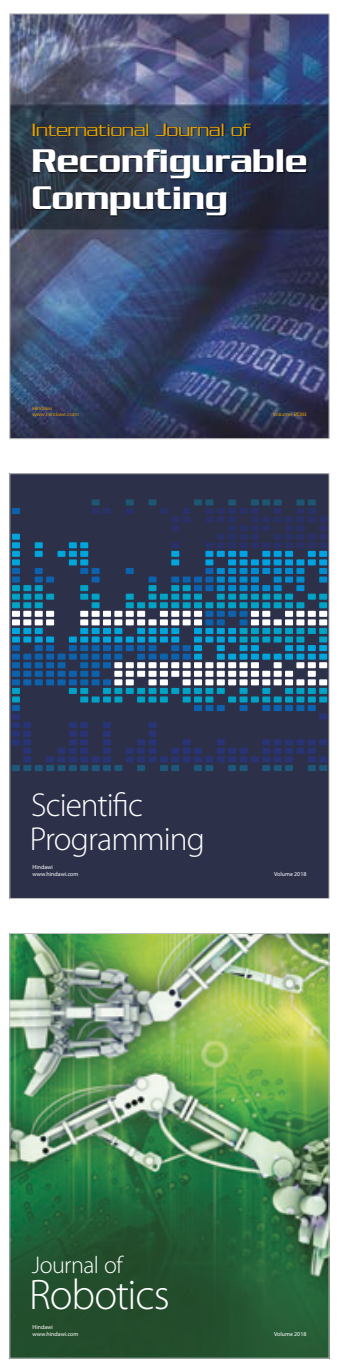

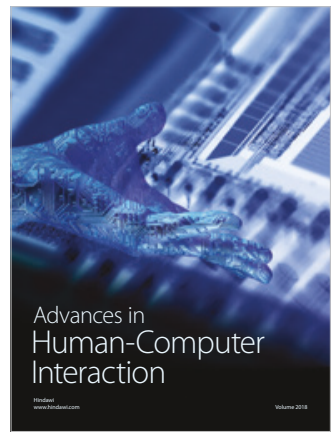

Human-Compute

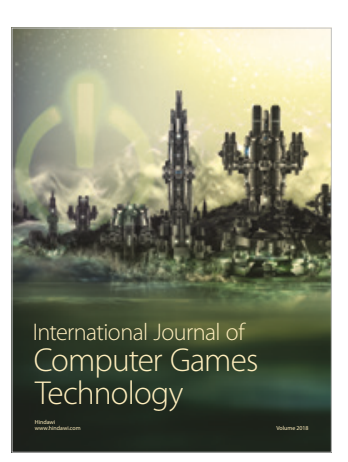

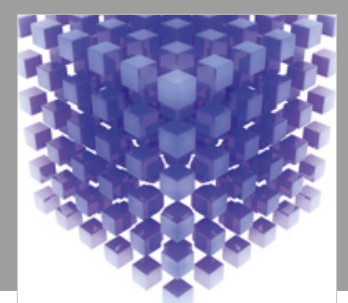

Mathematical Problems in Engineering

\section{Engincering}
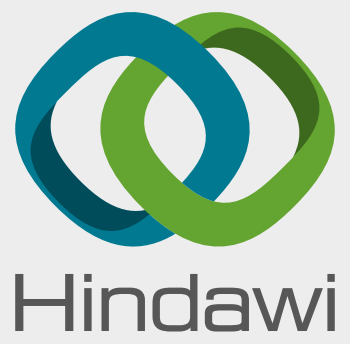

www.hindawi.com
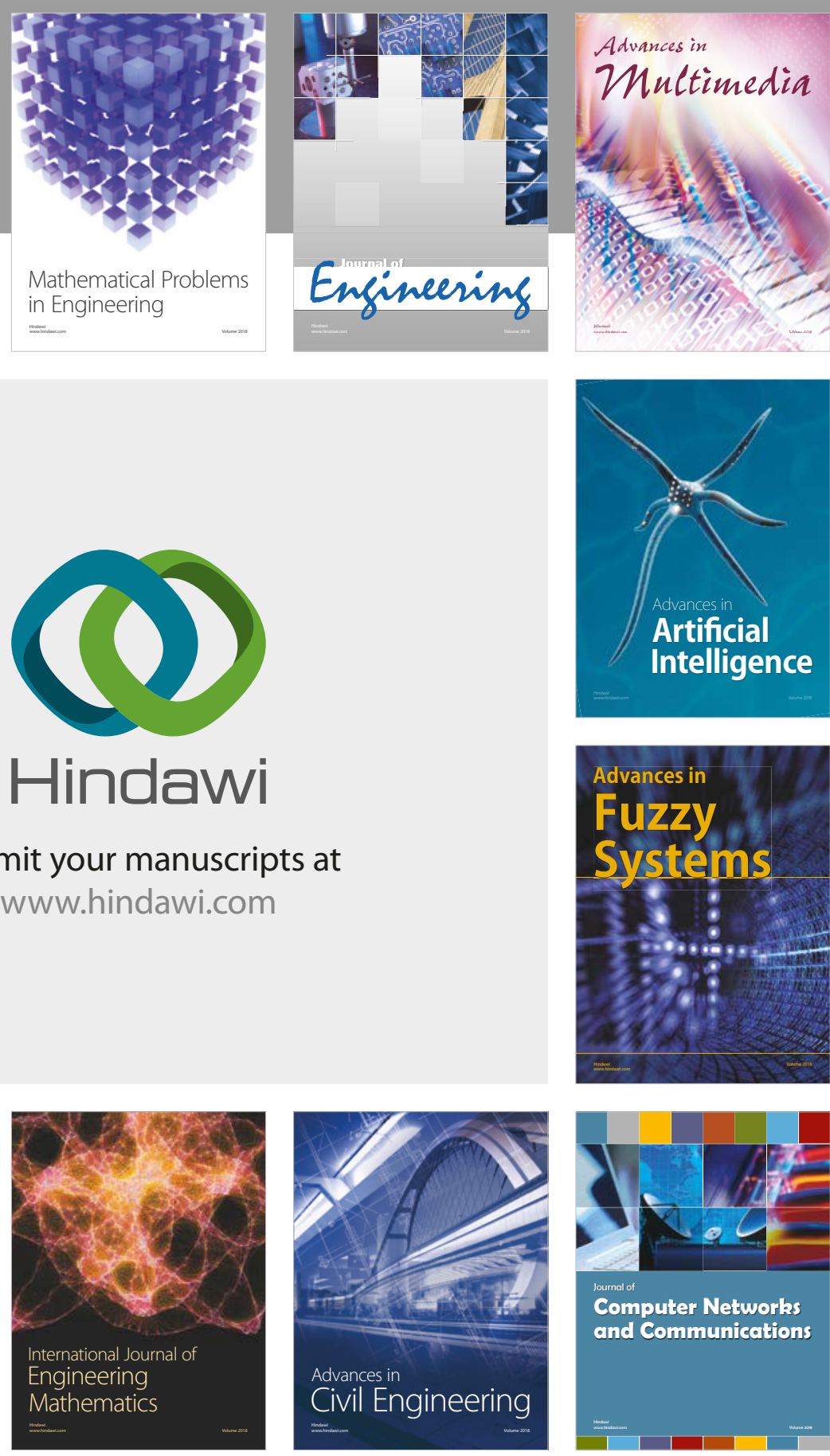

Computer Networks and Communications

Multimedia
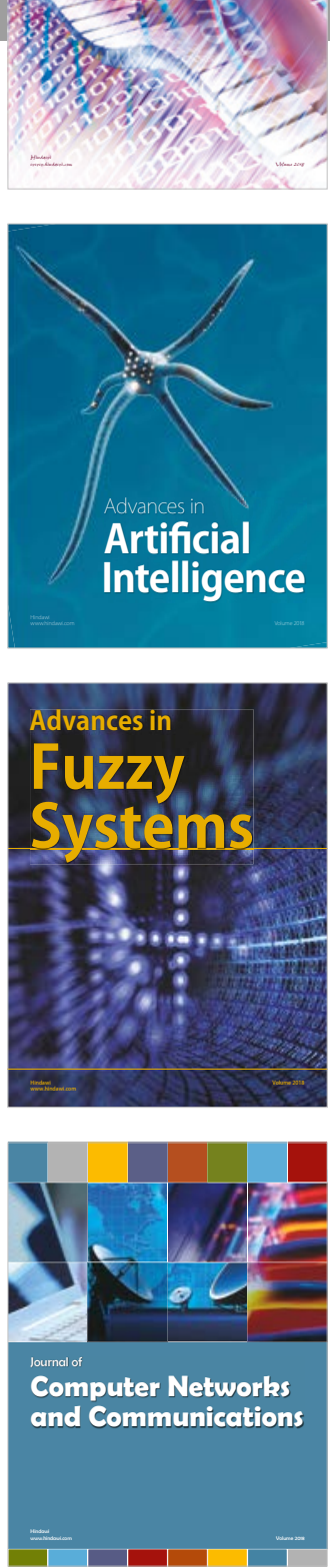

Advances in

Modelling \&

Simulation

in Engineering

interaction

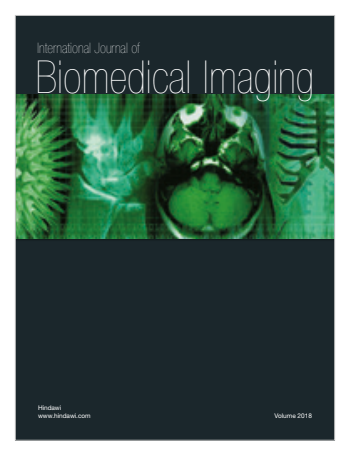

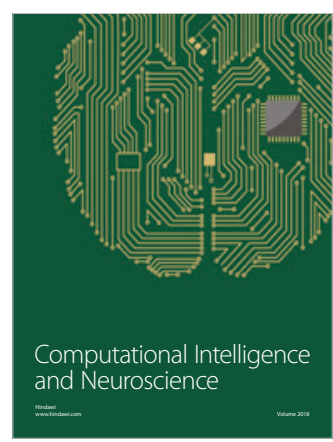

